\title{
Clima familiar, rendimiento académico e ingreso económico familiar en los estudiantes del VII ciclo de las áreas de internado de la Escuela de Psicología de la Universidad Cesar Vallejo 2006-I
}

Familiar atmosphere, academic performance and familiar economical income in VII cycle students from internship areas of Psychology school. Cesar Vallejo University. 2006-I

Carmen Rosa Tamayo Arteaga ${ }^{1}$.

\section{RESUMEN}

Objetivo: Establecer la relación entre clima social familiar, rendimiento académico e ingreso económico familiar en los estudiantes del VII ciclo de las áreas de internado de la Escuela de Psicología de la Universidad César Vallejo en el Semestre 2006-I. Materiales y Métodos: El estudio se realizó con un universo constituido por 91 estudiantes de las áreas de Internado (Clínica, Educativa y Organizacional). Para determinar el Clima Social en la Familia se empleó la escala propuesta por Moos y Trickett, la cual se aplicó a manera de encuesta. La validez del ensayo se determinó correlacionándola con la prueba de Bell. Para el análisis y comparación de items se utilizó el coeficiente de correlación de Pearson. La contrastación de hipótesis se realizó a través del Programa SPSS versión 14. Resultados: Se observó que el 63,7\% de estudiantes pertenece al área clínica, el $27,5 \%$ al área educativa y el 8,8\% al área organizacional. Por otro lado, el 81,32\% de los estudiantes pertenecen al rango de edad de 20 a 24 años, siendo el 64,84\% del género femenino, y en su mayoría solteros $(93,41 \%)$. Con respecto a sus ingresos económicos, el 68,13\% de los encuestados tiene un ingreso mensual familiar por encima de los mil soles, y el 50,55\% nacidos en la localidad de Trujillo. Finalmente, el promedio más alto de 53,35 pertenece a la dimensión desarrollo, el 52,64 a la dimensión relaciones y 41,54 a la dimensión estabilidad. Conclusión: No existe asociación significativa entre clima social familiar, rendimiento académico e ingreso económico familiar en los estudiantes encuestados.

Palabras clave: clima familiar, rendimiento académico, ingreso económico familiar, psicología.

\begin{abstract}
Objective: To establish a relationship between familiar atmosphere, academic performance and familiar economical income in VII cycle student of internship area from Psychology School of Cesar Vallejo University. Semester 2006-I. Materials and Methods: The study included a universe of 91 students from internship area (Clinics, Educative and Organizational). To evaluate the social atmosphere in the family, Moos and Trickett's scale was applied as a survey. The validity of this assay was determined by correlation with Bell's test. For analysis and comparison of items the Pearson's correlation coefficient was used. The hypothesis confirmation was performed in SPSS software version 14. Results: It was observed that $63,7 \%$ of student belongs to clinic area, $27,5 \%$ to educative area and $8,8 \%$ to organizational area. On the other hand, 81,32\% of students ranges from 20 to 24 years old, being $64,84 \%$ female, and most of them single $(93,41 \%)$. According to their incomes, $68,13 \%$ have incomes above one thousand soles, and 50,55\% were born in the location of Trujillo. Finally, the high average of 53,35 corresponds to the dimension development, 52,64 to the dimension relationships, and 41,54 to the dimension stability. Conclusion: There was no significant association between social familiar atmosphere, academic performance and familiar economical income between survey students.
\end{abstract}

Key words: familiar atmosphere, academic performance, familiar economical income, psychology.

\footnotetext{
${ }^{1}$ Magister em Docencia Universitaria. Universidad César Vallejo

Licenciada en Enfermería. Universidad Nacional de Trujillo
} 


\section{INTRODUCCIÓN}

El Clima Social Familiar se define como el conjunto de características psicosociales e institucionales de un determinado grupo asentado sobre un ambiente, lo que establece un paralelismo entre la personalidad del individuo y el ambiente (1). Asimismo, el Clima Social Familiar es el fruto de la suma de las aportaciones personales de cada miembro de la familia, traducidas en algo que proporciona emoción (2). En este sentido, el clima se enrarece o mejora en la medida en que se establecen relaciones entre dos personas, por lo que tendrían un papel decisivo las diferentes capacidades de sus miembros para establecer relaciones independientes de carácter dual y de convivencia de tipo grupal.

Con respecto al Rendimiento Académico éste se define como el resultado del proceso de interacción de enseñanza-aprendizaje, expresado en el logro de los objetivos educacionales. Es así que Olórtegui F (3), denomina Rendimiento Académico al nivel de conocimientos demostrados en una área $o$ materia comparado con la norma (edad y nivel académico). En este sentido, las calificaciones reflejarían las preferencias, dedicación y capacidad mayores o menores de los alumnos con relación a las distintas asignaturas. Por otro lado, encontramos que hay quienes homologan que rendimiento académico como el éxito o fracaso en el estudio expresado a través de notas o calificativos. Siguiendo esta definición, Rodríguez (4), refiere que el Rendimiento Académico es un indicador importante que manifiesta el grado de progreso del estudiante dentro del proceso de enseñanza-aprendizaje, para lo cual utiliza la calificación vigesimal (0-20) mediante la siguiente escala: BUENO (16-20), REGULAR $(11-15)$ y MALO $(<11)$. Dentro de los factores que estarían influyendo en el rendimiento académico se encuentra: el ambiente sociocultural, la política pedagógica, la familia y el alumno (5), pudiendo agruparse en endógenos (inteligencia y herencia) y exógenos (hábitos de estudio, ambiente de trabajo, etc.) (6).

En el caso del Ingreso Económico Familiar, el Instituto Nacional de Estadística e Informática (INEI), indica que el ingreso en soles (S/.) que tiene la familia, determina el nivel de pobreza y no pobreza; esto se manifiesta según lo citado por el Banco Interamericano de Desarrollo (BID), el cual emplea el método de la línea de pobreza usando como línea el costo por cápita, diario expresado en dólares.

Por este motivo, el presente trabajo tiene como objetivo establecer la relación entre clima social familiar, rendimiento académico e ingreso económico familiar en los estudiantes del VII ciclo de las áreas de internado de la Escuela de Psicología de la Universidad César Vallejo en el Semestre 2006-I.

\section{MATERIALES Y METODOS}

El universo estuvo constituido por 91 estudiantes del VII ciclo de las áreas de Internado (Clínica, Educativa y Organizacional) de la Escuela de Psicología de la Universidad César Vallejo en el Semestre 2006-I. Para determinar el Clima Social en la Familia se empleó la escala propuesta por Moos y Trickett (7), la cual se aplicó a manera de encuesta. La validez del ensayo se determinó correlacionándola con la prueba de Bell. Para el análisis y comparación de items se utilizó el coeficiente de correlación de Pearson. La contrastación de hipótesis se realizó a través del Programa SPSS versión 14.

\section{RESULTADOS}

Con respecto a los estudiantes del internado en Psicología de la UCV 2006-I, se observó que el $63,7 \%$ de los estudiantes pertenecen al área clínica, el 27,5\% al área educativa y un porcentaje menor de $8,8 \%$ al área organizacional.

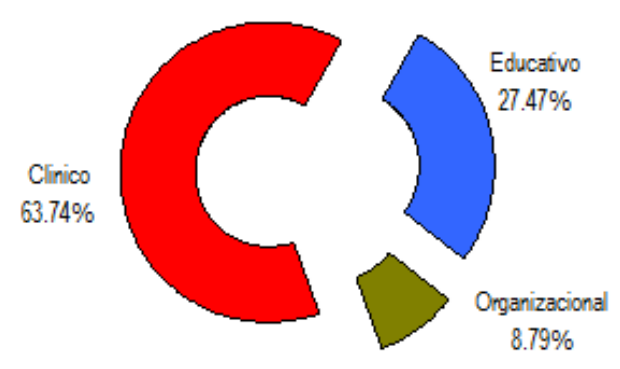

Fig. 1. Distribución de alumnos del internado en Psicología de la Universidad César Vallejo 2006-I 


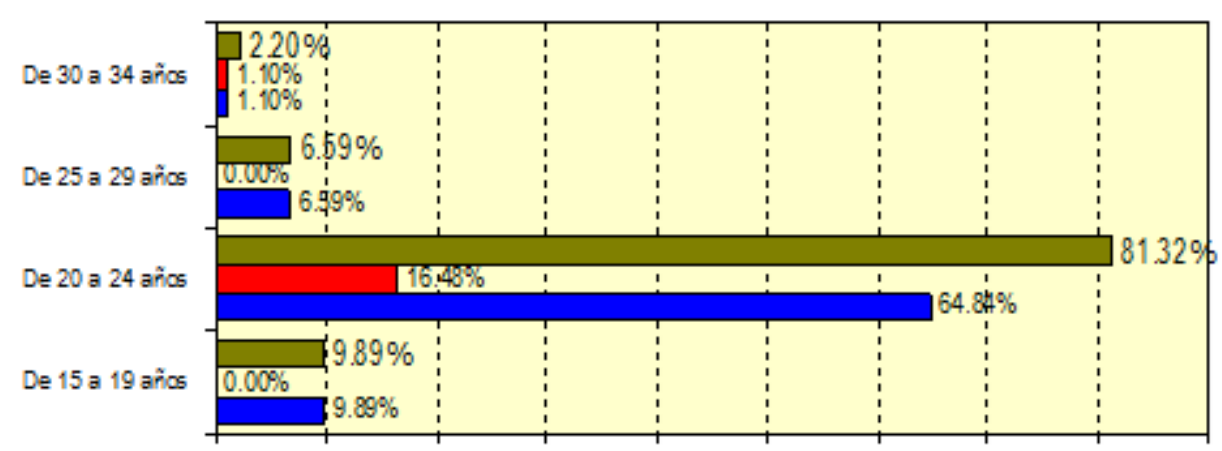

$0.00 \% \quad 10.00 \% 20.00 \% \quad 30.00 \% \quad 40.00 \% \quad 50.00 \% \quad 60.00 \% \quad 70.00 \% \quad 80.00 \% \quad 90.00 \%$

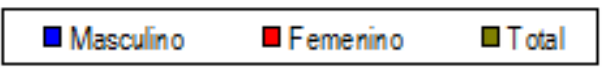

Fig. 2. Edad y género de los alumnos del internado en Psicología de la Universidad César Vallejo, Trujillo, 2006-I

En la Fig. 2 se observa que el $81,32 \%$ de la población de estudiantes encuestados que realizan dicho internado pertenecen al rango de edad de 20 a 24 años, de los cuales el $64.84 \%$ está representado por el género femenino, en tanto que el $16,48 \%$ pertenecen al género masculino, esto nos indica claramente que la relación de género femenino a masculino en este rango de edad es de 1 a 4 aprox. Asimismo se puede hallar que un porcentaje menor $(2,20 \%)$ de los estudiantes que realizan el internado en diferentes áreas se encuentra en el rango de edad de 30-34 años, y un porcentaje mínimo (9,89\%) entre 15-19 años y 6,59\% de rango de edad entre 25-29 años.

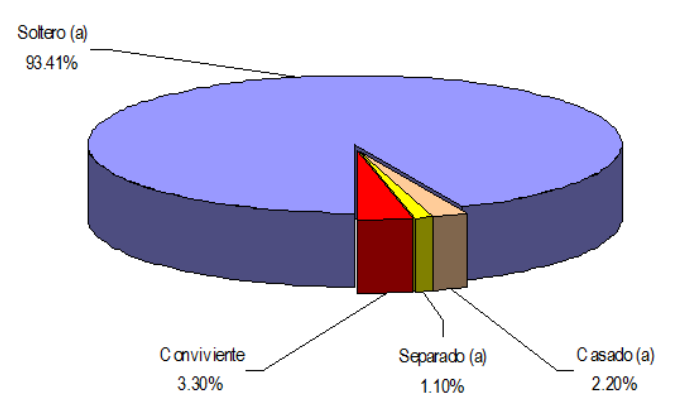

Fig. 3. Estado civil de los alumnos del internado en Psicología de la Universidad César Vallejo. Trujillo, 2006-I.

En la Fig. 3, se observa que los alumnos del internado de Psicología de la Universidad César Vallejo son en su mayoría solteros (93.41\%), otra parte son convivientes $(3.30 \%)$, casados $(2.20 \%)$ y separados $(1.10 \%)$. Estos resultados denotan también la fragilidad del hogar familiar con una incidencia de madres solteras (consideradas como "solteras") y convivientes de sus cónyuges.
Fig. 4. Ingreso económico de los hogares de los alumnos del internado en Psicología de la Universidad César Vallejo. Trujillo, 2006-I.

\begin{tabular}{lcc}
\hline \multicolumn{1}{c}{ Ingreso económico (S/.) } & $\mathbf{N}^{\mathbf{0}}$ & $\mathbf{\%}$ \\
\hline De 0 a 97,00 & 0 & 0.00 \\
Más de 97,00 hasta 500,00 & 6 & 6,59 \\
Más de 500,00 hasta 1000,00 & 23 & 25,27 \\
Más de 1000,00 & 62 & 68,13 \\
\hline TOTAL & $\mathbf{9 1}$ & $\mathbf{1 0 0 , 0 0}$ \\
\hline
\end{tabular}

Respecto al ingreso económico de los hogares de los alumnos del internado, la Fig. 4 nos muestra que no existe presencia de hogares en extrema pobreza, es decir familias con ingresos por debajo de 97 soles al mes (1 dólar diario), el 68,13\% de los hogares tiene un ingreso mensual familiar por encima de los mil soles, es decir el equivalente a 2 sueldos básicos, pero existe un $25,27 \%$ de los hogares que alcanza y supera un ingreso mínimo vital de 500,00 soles, pero menor a 2 sueldos básicos, y un escaso $6,59 \%$ o el equivalente a solo 6 familias tienen un ingreso superior a lo que se denomina pobreza, pero menor a un sueldo básico familiar.

En la Fig. 5, se aprecia que el 50,55\% de los alumnos del internado han nacido en la localidad de Trujillo; sin embargo es notoria la presencia de una emigración de familias proveniente de Cajamarca en un 9,89\%, porcentaje igual representan aquellos que han emigrado de las demás provincias de $\mathrm{La}$ Libertad, ya sea del Valle Liberteño o de la Sierra Liberteña. De igual manera podemos apreciar, que existe un porcentaje muy pequeño de emigrantes representados por alumnos provenientes de la parte Selva del Perú (Satipo, Iquitos) y del extranjero. 


\begin{tabular}{lcc}
\hline Lugar de Nacimiento & $\mathbf{N}^{\mathbf{0}}$ & $\%$ \\
\hline Cajamarca & 9 & $9.89 \%$ \\
Chiclayo & 6 & $6.59 \%$ \\
Chimbote & 4 & $4.40 \%$ \\
Iquitos & 1 & $1.10 \%$ \\
Lima & 2 & $2.20 \%$ \\
Piura & 7 & $7.69 \%$ \\
Otros Prov. de la Libertad & 9 & $9.89 \%$ \\
Satipo & 1 & $1.10 \%$ \\
Tarapoto & 2 & $2.20 \%$ \\
Trujillo & 46 & $50.55 \%$ \\
Tumbes & 3 & $3.30 \%$ \\
Extranjero & 1 & $1.10 \%$ \\
\hline TOTAL & $\mathbf{9 1}$ & $\mathbf{1 0 0 . 0 0 \%}$ \\
\hline
\end{tabular}

Fig. 5. Lugar de nacimiento de los alumnos del internado en Psicología de la Universidad César Vallejo. Trujillo, 2006-I.

En la Fig. 6, se muestran los promedios de clasificación de alumnos en diferentes dimensiones. El promedio más alto es 53,35, perteneciente a la dimensión desarrollo, en tanto que en las dimensiones relaciones y estabilidad obtuvieron 52,64 y $\quad 41,54$ respectivamente.

\begin{tabular}{lc}
\hline \multicolumn{1}{c}{ Dimensión } & Promedio \\
\hline Relaciones & 52,64 \\
Desarrollo & 53,35 \\
Estabilidad & 41,54
\end{tabular}

Fig. 6. Alumnos del internado en Psicología de la Universidad César Vallejo, según promedio en las dimensiones del Clima Social Familiar. Trujillo, 2006I.

\section{DISCUSIÓN}

Los resultados que se han obtenido en este estudio luego de aplicar la Escala de Clima Social en la Familia (FES) de R.H, Moos y E.J. Trickett permite mencionar en términos generales que no se encuentra correlación estadísticamente significativa entre las variables de estudio y los estudiantes que realizan su internado en diferentes áreas (clínica, educativa y organizacional), lo cual indica que de una u otra manera el clima social familiar es la apreciación de las características socio-ambientales de la familia, la misma que es descrita en función de las relaciones interpersonales de los miembros de la familia, los aspectos de desarrollo que tienen mayor importancia en ella y su estructura básica; es decir la dinámica familiar organizada facilita la reducción del estrés en el hogar, contribuyendo incluso al éxito académico y personal o caso contrario a que la persona cuente con recursos personales para alcanzar objetivos trazados. Esto es mencionado en otras referencias (8) en el sentido de que es totalmente independiente el ingreso económico familiar ya que la mayoría de los hogares $(68.13 \%)$ tienen un ingreso mensual familiar por encima de los 1000 soles; es decir el equivalente a dos sueldos básicos y pueden decidir qué hacer con su ganancia económica en diversas áreas de su vida (Fig. 4). Es importante resaltar que el clima social familiar es el fruto de la suma de las aportaciones personales de cada miembro de la familia y es el ambiente de interacción que coadyuva al desarrollo y adaptación personal de sus miembros; asimismo permite establecer relaciones independientes de carácter dual y de convivencia de tipo grupal, en este sentido se puede estar de acuerdo con varios autores (9) que confirman lo anteriormente dicho.

Con respecto al rendimiento académico, Aliaga (10) menciona que el rendimiento académico es el resultado del proceso de interacción enseñanza-aprendizaje. Asimismo Angles (6) afirma que no se encuentra un mejor criterio de medida del rendimiento académico que el de las calificaciones de los profesores a los alumnos mediante el proceso de evaluación. Todo esto es confirmado por Rodríguez Rivera (1994) el cual considera que el promedio de aprendizaje es utilizando la calificación vigesimal (0-20) lo cual se expresa: Bueno (16-20), Regular (11-15) y Malo $(<11)$. Asimismo, respecto al ingreso económico familiar es importante mencionar a 
Mizala (11) que refiere que los padres de familia pueden promover un alto rendimiento en sus hijos, no importa donde vivan o cuales sean sus ingresos económicos, si hacen saber a sus hijos que tienen altas expectativas en ellos. Los padres pueden transmitirles la importancia del bajo rendimiento y cuáles son sus consecuencias, así como también el alto rendimiento y aumentar la autoestima de sus hijos, estableciendo metas a corto plazo y proporcionando recompensas cuando sus hijos alcancen esas metas. Por otro lado, Angles (6) considera que las familias deciden las instituciones educativas públicas o privadas para que sus hijos reciban educación según su nivel de ingreso; es decir se puede apreciar que los hogares incrementan la proporción que destinan a la educación según aumenta su nivel económico, se puede decir que está en concordancia con el concepto dado por Albarello (12).

En general, se puede decir que el grado de las dimensiones: relaciones, desarrollo y estabilidad entre los miembros del hogar, aquellos que brindan un clima de participación entre sus integrantes permiten la expresión libre y espontánea, manejan con serenidad sus conflictos, son más independientes y toman sus decisiones; en contraposición de aquellos hogares que viven un estilo de vida no saludable con respecto a lo anteriormente mencionado y aun teniendo en cuenta que las relaciones al interior del hogar por parte de sus miembros puede ser un aspecto importante para el desarrollo personal de todos, el rendimiento académico es independiente de este factor. Estos resultados coinciden con lo reportado por previamente, donde se confirma que el desempeño académico de los estudiantes es independiente del clima social familiar en sus dimensiones: relaciones, desarrollo, estabilidad $(8,9)$.

\section{CONCLUSIONES}

No existe asociación significativa entre clima social familiar, rendimiento académico e ingreso económico familiar en los estudiantes del VII ciclo de las áreas de internado de la Universidad César Vallejo en el Semestre 2006-I. Asimismo se determina que no existe una asociación significativa entre el ingreso económico y el rendimiento académico.

\section{AUTOR DE CORRESPONDENCIA}

\author{
Mg. Carmen Rosa Tamayo Arteaga \\ Universidad César Vallejo \\ Trujillo - Perú \\ Teléfono: 975519936 \\ E-mail: carmentamayoa@gmail.com
}

\section{REFERENCIAS BIBLIOGRÁFICAS}

1. Horna Carranza A. (1997). Psicología médica. Ed. Libertad. Trujillo. 105-112 pp.

2. Mizala A et al. Factores Sociales y Económicos de las Familias. Universidad de Chile.106-123pp.

3. Olórtegui Miranda F. (2002) Problemas de aprendizaje. Ed. San Marcos. Lima. 121-128 pp.

4. Rodriguez Rivera V. (1994) Psicotécnica Pedagógica. Teoría-Práctica. Ed. Trillas. México.111-118 pp.

5. Pomalina Rodriguez. (1998) Salud mental del escolar. Fracaso escolar. Manual de referencia. Lima. 35-57 pp.

6. Angles C (1983). Evaluación del rendimiento escolar. Ed. Universo S.A. Lima, 89-94 pp.

7. Moos RH, Trickett (1985). Escala del clima social familiar. Madrid. TEA Ediciones.

8. Freyre Román E. (1994). La salud del adolescente. Lima.87-95pp.

9. Rodríguez Rivera V (1994) Psicotécnica Pedagógica. Teoría- Práctica. Ed. Trillas. México. 111-118 pp.

10. Aliaga J. La ubicación espontánea del asiento en el aula como función de la inteligencia, la personalidad, el rendimiento académico y el sexo. Revista de Psicología. Volumen 4. 1999 /Diciembre.87-92pp.

11. Mizala A et al. (2000). Factores Sociales y Económicos de las Familias. Universidad de Chile. 106-123 pp.

12. Albarello F. Espacios de Comunicación Alternativa y Nuevas Tecnologías. La Brecha Digital y su Abordaje Educativo. Revista de Psicología. Volumen 2 .2004/ Junio.75-80pp.

Recibido: 02/09/2016

Aceptado: 11/11/2016 